\title{
ONE EXAMPLE OF THE BOUNDARY BEHAVIOUR OF BIHOLOMORPHIC TRANSFORMATIONS
}

\author{
B. L. FRIDMAN
}

\begin{abstract}
Two biholomorphically equivalent domains $\Omega_{1}, \Omega_{2} \subset \mathbf{C}^{2}$ with piecewise smooth boundaries and with the following property are constructed. If $F: \Omega_{1} \rightarrow \Omega_{2}$ is any biholomorphic transformation then neither $F$ nor $F^{-1}$ can be extended continuously to the boundary.
\end{abstract}

0. In the theory of functions of one complex variable the following statement (Carathéodory theorem) is true. Let $f: \Omega_{1} \rightarrow \Omega_{2}$ be a biholomorphic mapping, $\Omega_{1}$, $\Omega_{2} \subset \mathbf{C}^{1}$ are domains bounded by piecewise smooth simple closed curves. Then $f$ admits a continuous extension up to the boundary $\partial \Omega_{1}$ of $\Omega_{1}$. In $\mathbf{C}^{n}, n>1$, for some types of domains (say, some types of pseudoconvex domains with smooth boundaries or analytic polyhedra, see [1-6]) an analogous theorem is true. But actually in $\mathbf{C}^{n}$, $n>1$, the situation is different (for further discussions see [7]).

The purpose of this paper is to construct two bounded domains $\Omega_{1}, \Omega_{2} \subset \mathbf{C}^{2}$ with the following properties.

$\left(\mathrm{P}_{1}\right) \Omega_{1}$ and $\Omega_{2}$ have piecewise smooth boundaries homeomorphic to the sphere $\partial B^{2}=\left\{\left.z \in \mathbf{C}^{2}|| z_{1}\right|^{2}+\left|z_{2}\right|^{2}=1\right\}$.

$\left(\mathrm{P}_{2}\right)$ Both $\Omega_{1}$ and $\Omega_{2}$ are biholomorphically equivalent to the polydisc $D^{2}=\{z \in$ $\left.\mathrm{C}^{2}|| z_{j} \mid<1, j=1,2\right\}$.

$\left(\mathrm{P}_{3}\right)$ There exists a $C^{\infty}$ one-to-one mapping $f: \bar{\Omega}_{1} \rightarrow \bar{\Omega}_{2}(\bar{\Omega}$ is the closure of $\Omega)$.

$\left(\mathrm{P}_{4}\right)$ For any biholomorphic mapping $F: \Omega_{1} \rightarrow \Omega_{2}$ neither $F$ nor $F^{-1}$ can be extended continuously to the boundary.

REMARK. The family $\Phi$ of such biholomorphic mappings is quite large. If $G_{k}: D^{2} \rightarrow \Omega_{k}, k=1,2$, are fixed biholomorphic mappings, then $\Phi=$ $\left\{G_{2} \circ G \circ G_{1}^{-1} \mid G \in\right.$ Aut $\left.D^{2}\right\}$, where Aut $D^{2}$ is the group of holomorphic automorphisms of $D^{2}$. So, $\Phi$ is as "large" as Aut $D^{2}$ which is a 6-parameter group (for description of Aut $D^{2}$ see [7]).

1. Definition of $\Omega_{k}, k=1,2$.

(A) First we choose two sets of points $\zeta_{s k} \in \mathbf{C}, s=1,2,3,4, k=1,2$, with the following properties:

$\left(\mathrm{A}_{1}\right)\left|\zeta_{s k}\right|=1$ for all $s, k$.

$\left(\mathrm{A}_{2}\right)$ There does not exist a biholomorphic automorphism $g$ of the unit disc, $g: D \rightarrow D$, such that $g\left(\cup_{s=1}^{4} \zeta_{s 1}\right)=\cup_{s=1}^{4} \zeta_{s 2}$. 
Remark. Every $g \in$ Aut $D$ can be extended analytically to the boundary $\partial D$. So, in fact, in $\left(\mathrm{A}_{2}\right) g: \bar{D} \rightarrow \bar{D}$.

It is easy to see that such points $\left\{\zeta_{s k}\right\}$ exist.

(B) Consider now two mappings $F_{k}: D^{2} \rightarrow \mathrm{C}^{2}$;

$$
F_{k}:\left\{\begin{array}{l}
w_{1}=z_{1}, \\
w_{2}=z_{2} \prod_{s=1}^{4}\left(\zeta_{s k}-z_{1}\right), \quad k=1,2 .
\end{array}\right.
$$

We set now $\Omega_{k}=F_{k}\left(D^{2}\right), k=1,2$.

2. TheOREM. $\Omega_{k}, k=1,2$, have properties $\left(\mathrm{P}_{1}\right)-\left(\mathrm{P}_{4}\right)$.

Proof. (a) $\left(\mathrm{P}_{1}\right)$ and $\left(\mathrm{P}_{2}\right)$ are almost obvious and follow from the description of $F_{k}$. (b) To prove $\left(\mathrm{P}_{3}\right)$ let us take a $C^{\infty}$ mapping $g: \bar{D} \rightarrow \bar{D}$ which is one-to-one and such that $g\left(\cup_{k=1}^{4} \zeta_{k 1}\right)=\cup_{k=1}^{4} \zeta_{k 2}$. We set $G:\left(z_{1}, z_{2}\right) \rightarrow\left(g\left(z_{1}\right), z_{2}\right)$. Then the mapping $F_{2} \circ G \circ F_{1}^{-1}: \Omega_{1} \rightarrow \Omega_{2}$ can be extended to a $C^{\infty}$ one-to-one mapping $\bar{\Omega}_{1} \rightarrow$ $\bar{\Omega}_{2}$.

(c) Now we check property $\left(\mathrm{P}_{4}\right)$. Let $F: \Omega_{1} \rightarrow \Omega_{2}$ be a biholomorphic mapping. Let us consider $G=F_{2}^{-1} \circ F \circ F_{1}: D^{2} \rightarrow D^{2}$. Evidently $G \in$ Aut $D^{2}$. From the description of this group $G=\left(g_{1}, g_{2}\right)$, where $g_{k} \in$ Aut $D, k=1,2$, and either $g_{1}=g_{1}\left(z_{1}\right), g_{2}=g_{2}\left(z_{2}\right)$ or $g_{1}=g_{1}\left(z_{2}\right), g_{2}=g_{2}\left(z_{1}\right)$. Let $g=g\left(z_{1}\right)$ denote $g_{1}$ if we are dealing with the first case, and $g_{2}$ otherwise. Since property $\left(\mathrm{A}_{2}\right)$ holds for $\left\{\zeta_{s k}\right\}$ we can always find an integer $j, 1 \leqslant j \leqslant 4$, such that

$$
g\left(\zeta_{j 1}\right) \neq \zeta_{s 2} \text { for any } s, 1 \leqslant s \leqslant 4 \text {. }
$$

Let us now take a sequence $\left\{\lambda_{n}\right\}_{n=1}^{\infty} \subset D$ such that $\lim _{n \rightarrow \infty} \lambda_{n}=\zeta_{j 1}$, and consider two sequences $u_{n}=F_{1}\left(\lambda_{n}, \frac{1}{2}\right)$ and $v_{n}=F_{1}\left(\lambda_{n},-\frac{1}{2}\right), 1 \leqslant n<\infty$. Both of them lie in $\Omega_{1}$ and, according to (1),

$$
\lim _{n \rightarrow \infty} u_{n}=\lim _{n \rightarrow \infty} v_{n}=\left(\zeta_{j 1}, 0\right) \in \partial \Omega_{1} .
$$

On the other hand, in the first case $\left(g=g_{1}\right)$ we have

$$
\lim _{n \rightarrow \infty} F\left(u_{n}\right)=\lim _{n \rightarrow \infty}\left(F_{2} \circ G \circ F_{1}^{-1}\right)\left(u_{n}\right)=F_{2}\left(g\left(\zeta_{j 1}\right), g_{2}\left(\frac{1}{2}\right)\right)
$$

and

$$
\lim _{n \rightarrow \infty} F\left(u_{n}\right)=F_{2}\left(g\left(\zeta_{j 1}\right), g_{2}\left(-\frac{1}{2}\right)\right) .
$$

From (2) and the description of $F_{2}$,

$$
\lim _{n \rightarrow \infty} F\left(u_{n}\right) \neq \lim _{n \rightarrow \infty} F\left(v_{n}\right) \text {. }
$$

The second case $\left(g=g_{2}\right)$ can be considered similarly. In both cases (4) holds. $\left(\mathrm{P}_{4}\right)$ now follows from (3), (4) and similar considerations for $F^{-1}$.

\section{REFERENCES}

1. S. Bell, Smooth bounded strictly and weakly pseudoconvex domains cannot be biholomorphic, Bull. Amer. Math. Soc. (N.S.) 4 (1981), 119-120.

2. S. Bell and D. Cathlin, Proper holomorphic mappings extend smoothly to the boundary, Bull. Amer. Math. Soc. (N.S.) 7 (1982), 269-272. 
3. K. Diederich and J. E. Fornaess, Smooth extendability of proper holomorphic mappings, Bull. Amer. Math. Soc. (N.S.) 7 (1982), 264-268.

4. C. Fefferman, The Bergman kernel and biholomorphic mappings of pseudoconvex domains, Invent. Math. 26 (1974), 1-65.

5. B. L. Fridman, On a class of analytic polyhedra, Dokl. Akad. Nauk SSSR 242 (1978); English transl., Soviet Math. Dokl. 19 (1979), 1258-1261.

6. G. Henkin, An analytic polyhedron is not holomorphically equivalent to a strictly pseudoconvex domain, Soviet. Math. Dokl. 14 (1973), 858-862.

7. S. G. Krantz, Function theory of several complex variables, Wiley, New York, 1982.

Department of Mathematics, Wichita State University, Wichita, KanSas 67208 